\title{
Jejunum free flap in hypopharynx reconstruction: Case series
} Marco Benazzo*1, Antonio Occhini ${ }^{1}$, Vanessa Rossi ${ }^{1}$, Giuseppe Aresi ${ }^{1}$ and Mario Alessiani ${ }^{2}$

Address: ${ }^{1}$ Dept. of Otorhinolaryngology, University of Pavia, IRCCS Policlinico S. Matteo, Pavia, Italy and ${ }^{2}$ Hepatopancreatic Surg. Unit, University of Pavia, IRCCS Policlinico S. Matteo, Pavia, Italy

E-mail: Marco Benazzo* - m.benazzo@smatteo.pv.it; Antonio Occhini - m.benazzo@smatteo.pv.it; Vanessa Rossi - vrossiorl@hotmail.com; Giuseppe Aresi - giaresi@yahoo.it; Mario Alessiani - m.alessiani@unipv.it

${ }^{*}$ Corresponding author

This article is available from: http://www.biomedcentral.com/ I47/-2407/2//3

(c) 2002 Benazzo et al; licensee BioMed Central Ltd. Verbatim copying and redistribution of this article are permitted in any medium for any purpose, provided this notice is preserved along with the article's original URL.

\begin{abstract}
Background: Surgical treatment of hypopharyngeal cancers with extension to the retrocricoid region generally requires a circumferential pharyngolaryngectomy followed by a reconstruction of the removed segment of the upper digestive tract. Historically, many techniques have been used in order to achieve a safe and functional reconstruction. Jejunum interposition is generally considered the best reconstructive technique.
\end{abstract}

Methods: This study examines the details of the surgical technique, the complications, the oncological and the functional results in a series of 29 consecutive patients submitted to circumferential pharyngoesophageal resection for advanced hypopharyngeal cancer followed by reconstruction with a free flap of jejunum.

Results: Three of the transplants failed because of venous thrombosis. The overall success rate was $90 \%$. There were no general complications. A good swallowing has been preserved in all our patients. All our patients where a phonatory prosthesis was positioned (20/29) were able to achieve speech following speech therapy and all were satisfied with their own capacity to communicate.

Conclusions: The prognosis of hypopharyngeal tumours ( $18-40 \%$ at 5 years) remains poor, but jejunum autografts are being shown to be an excellent choice for the reconstruction of the cervical hypopharyngo-oesophagus offering the patient fast rehabilitation and a reasonable quality of survival. Our experience confirm that this kind of reconstruction is safe with a good results in improving oncologic controls and restoring a good quality of life.

\section{Background}

Extended hypopharyngeal tumors are an aggressive disease with a poor prognosis irrespective of the therapeutic regimen instituted. The 5-year survival rate in patients with stage III and IV disease is no greater than $18-40 \%$ $[1,2]$. Incase of extension of retriocricoid region or the posterior wall with involvement of both piriform sinuses surgical treatment generally requires a circumferential pharyngolaryngectomy followed by a reconstruction of the removed segment of the upper digestive tract. 
Historically many techniques have been used in order to achieve a safe and functional reconstruction [3-5]. Free jejunum transfer and gastric pull-up are two modalities that have become particularly accepted [6-11]. Jejunum interposition is generally considered the best reconstructive technique when the distal neoplastic margin does not macroscopically extend more than $2 \mathrm{~cm}$ below the inferior bord of cricoid [12-15]. This technique has gained the attention of head and neck surgeons because of its undoubted anatomical and physiological advantages, requiring the sacrifice of only a short section of bowel, which anatomically adapts well to the segment to be reconstructed and guarantees a fast functional recovery and an acceptable quality of life $[12,16]$.

This study examines the details of the surgical technique, the complications, the oncological and the functional results in a series of 29 consecutive patients submitted to circumferential pharyngoesophageal resection for advanced hypopharyngeal cancer followed by reconstruction with a free flap of jejunum.

\section{Methods}

From January 1996 to December 2000, 29 patients (27 males, 2 females) aged between 45 and 73 years (mean 57.1, median 57) admitted to our department underwent circular resection of the hypopharynx and reconstruction with a revascularized free flap of jejunum. In 2 cases we performed a double flap (jejunum + pectoralis major) for oncological reasons. Of these patients, 19 had no previous treatment, 2 were treated for another head and neck tumor ( 1 oropharyngeal, 1 oral cavity). Of the remaining 8 patients, 4 were treated with chemo-radiotherapy treatment for laryngeal or hypopharyngeal tumors and 4 were post-operative recurrences. Of these last four patients treated for laryngeal cancer, 1 had a peristomal recurrence, 1 had recurrence at the junction between the pharyngeal mucosa and the previously transplanted pectoralis major muscle, and 2 had recurrences on the posterior wall of the neopharynx. All these 10 patients had previously radio- therapy. The histological diagnosis was squamous cell carcinoma in all cases. The patients' TNM staging is reported in Table 1. The staging procedure included panendoscopy of the upper respiratory and digestive tracts, and CT scan and/or MRI of the neck and mediastinum. All patients were included in a protocol for the evaluation of metabolic, nutritional and cardiovascular status. The Karnofski index ranged between 90 and 100 for all the patients. A Doppler ultrasonography examination of the supra-aortic branches was carried out in all patients.

As far as concern functional results, we evaluated swallowing by a swallowing questionnaire (SQ) and voice quality (intelligibility, pleasantness and comprehension) by a computerized voice analysis (KAY CSL 4300) system. All subjects were first asked to pronounce the vowel /a/ at an intensity they were comfortable with and to hold it for an acceptable lenght of time. We evaluated the spectrographic features of the signal, the fundamental frequency (F0) and the parameters of waveform perturbations such as percentual jitter (\%), absolute shimmer (dB) and harmonic to noise ratio (HNR). The measurements were performed on a central segment of approximatively 2 seconds of the sustained voice.

The spectrographic analysis of the italian word "aiuole" (english: flower beds), that contains all the five vowels, was then made to analyse the formants. Maximum phonation time (MPT) was calculated asking each patient to produce the vowel /a/ as long as possible at a comfortable level of intensity for three times. The best of the 3 trials was chosen.

Qualitative evaluation of speech was based on the following voice parameters: intelligibility, pleasantness and acceptability. By vocal intelligibility we mean the ability for the listener to fully understand a verbal message. The term pleasantness is used to indicate a subjective evaluation of vocal esthetics. By acceptability we mean an overall judgement taking into account both these parameters.

Table I: TNM classification

\begin{tabular}{|c|c|c|c|c|c|c|c|}
\hline & No & NI & $\mathrm{N} 2 \mathrm{a}$ & $\mathrm{N} 2 \mathrm{~b}$ & $\mathrm{~N} 2 \mathrm{c}$ & N3 & TOTAL \\
\hline TI & & & & & & & 0 \\
\hline $\mathrm{T} 2$ & 4 & 3 & & & & & 7 \\
\hline T3 & 6 & 1 & & & & & 7 \\
\hline $\mathrm{T} 4$ & 9 & 0 & 0 & 3 & 2 & I & 15 \\
\hline TOTAL & 19 & 4 & 0 & 3 & 2 & 1 & 29 \\
\hline
\end{tabular}


Both evaluations were performed at three and six months postoperatively. Weight was assessed at the same intervals.

Concerning the surgical technique the operation was carried out by two surgical teams. No bowel preparation was required before surgery. The abdominal stage started once the cervical dissections were carried out. While the cervical team carried out the pharyngeal circular resection, the second team carried out a median laparotomoy and selected the section of jejunum with appropriate vasculature for the preparation of a long vascular mesenteric pedicle. In most cases the third vascular segment was the most suitable in that it is supplied by a large, constant vascular pedicle. The segment was sectioned with preservation of the remaining jejunal vascularization, using the transillumination technique to ensure vascularization of the remaining jejunum through the vascular arcades in the mesentery. Subsequently a termino-terminal anastomosis of the digestive tract was fashioned. The pedicle of the jejunal segment was not excised until the end of the resection of the tumour and the preparation of the recipient cervical vessels, preceded by the intravenous administration of 1500 IU heparin sulphate.

We normally carried out the vascular anastomoses first, in order to reduce the time that the transplant is ischemic, and normally the venous anastomosis before the arterial one. In most cases the arterial anastomosis was made with the superior thyroid artery, which is particularly suitable as far as regards size and spatial orientation. The venous anastomosis was made with the internal jugular vein or the tiro-lingual trunk. Particular care was given to positioning the loop in an isoperistaltic direction in order to facilitate the subsequent passage of food and avoid persistent dysphagia. The perfusion of the flap was assessed and confirmed by the almost immediate return of peristalsis, by the visceral secretion in the jejunum loop, by the bleeding of the segment and by the restoration of the shiny pink colour of the serosa. As a precaution a minimal jejunostomy was created in all the patients until the natural route of nutrition was restored [17]. Antibiotic prophylaxis with amoxicillin-clavulanic acid (1 g. i.v.) was started at the induction of anaesthesia and continued every four hours for 24 hours. The patients were monitored during the anaesthesia through an arterial cannula and kept warm with a termal blanket. The analgesic therapy was started at the end of the surgical operation by administering $10 \mathrm{mg}$ of morphine that could be repeated after six hours. Diuresis was monitored and haematological an biochemical examinations were performed every 12 hours.

Flap viability was monitored in 17 patients through an anterior neck window, closed on the third day; in the other
12 patients the monitoring took place via direct observation of a segment of loop positioned externally to the surgical wall and removed on the fifth post-operative day. From the third day onwards, the patients underwent daily endoscopic examination of the flap. Anticoagulation treatment with low molecular weight heparin (fraxiparin 2850 IU once a day) was started in all patients on the first day post-operatively and continued for thirty days.

Three days after surgery the patients started to swallow a vancomicin antibiotic solution (500 $\mathrm{mg} \times 3$ each day) in order to sterilize the mucosal sutures. A gastrografin swallow was carried out between 7-10 days post-operatively, and once dehiscence of the sutured mucosa had been excluded, oral alimentation was recommenced, first with liquids and then with semisolids for the first 15 days. From the 15th day, a full range of food was allowed.

In 15 patients who had adjuvant radiotherapy, the gastrostomy was retained as a precaution until the end of the RT.

In 10 patients we placed a vocal prosthesis (Provox type) with a fistula at the level of tracheotomy, between the posterior wall of the trachea and the anterior wall of the jejunum. This operation was performed 6-12 months after the oncological surgery and in no case earlier than three months after the end of post-operative radiation treatment.

\section{Results}

The mean duration of the operation was 8 hours (5-12 hours). There were no surgically related post-operative deaths. Three of the transplants failed because of venous thrombosis on days 5,7 and 30. In one case the necrotic flap diagnosis of was early enough to allow replacement of the necrotic loop with another, while in the other two cases it was necessary to reconstruct the digestive tract with a pedicled myocutaneous flap of tubulized pectoralis major muscle [12]. The overall success rate was $90 \%$.

There were no general complications. As far as concerns complications of the transplant site, excluding the cases of necrosis of the flap, one salivary fistula developed due to infection in the neck. This was treated successfully by a covering with a pedicled flap of deltopectoralis muscle. As far as concerns the donor site, one patient had a haemorrhage at the site of the abdominal surgery which required revision (Table 2).

\section{Functional results}

Overall the nasogastric tube was removed after a mean of 15 days (range 9-150). In 25 patients was removed before the 15 th day. 
Table 2: General, local and donor site complications.

\begin{tabular}{llccc}
\hline & & $\begin{array}{c}\text { No. of } \% \\
\text { patients }\end{array}$ \\
\hline \multirow{2}{*}{ GENERAL } & & & & \\
& Early & Flap necrosis & none & \\
& & Salivary fistula & 2 & $10.34 \%$ \\
LOCAL & & 1 & $3.4 \%$ \\
& Late & Flap necrosis & & \\
& & Neck cellulitis & 1 & $3.4 \%$ \\
DONOR SITE & & m Bowel haemorrhage & I & $3.4 \%$ \\
& & & & \\
\hline
\end{tabular}

Early: before 15 days Late: after 16 days

At three months after the operation only one patient presented difficulty to assume a solid diet and 3 a liquid diet. Six months after all the living patients were able to eat a reasonably normal diet and none of them has a gastrostomy. Also in the fifteen patients who received adjuvant radiotherapy, which was tolerated extremely well, the swallowing function had shown no impairment.

Voice quality (intelligibility, pleasantness, and comprehension) was unsatisfactory in all patients except the 10 patients where a voice prosthesis was positioned in which the functional results are excellent. There were no complications in the positioning of the prosthesis, or in its longterm functional results. No patient had post-operative complications due to the insertion of the voice button. No patient had to have the prosthesis removed during the follow-up ( 8 to 14 months). All the patients were able to achieve voice following the tracheojejunum puncture and were satisfied with their own capacity to communicate thank to the specific intensive rehabilitation program.

\section{Oncological results}

The surgical resection margins were uninvolved by the tumor in 28 cases $(96.5 \%)$ and invaded in 1 cases (3.5\%).

The neck lymphnodes were removed from 27 patients, of whom 19 had bilateral modified radical neck dissection (MRND type III), 3 selective bilateral (levels II,III,IV) and 3 monolateral MRND. In 2 patients a bilateral excision was performed, radical on one side and MRND on the other. We treated 48 necks. The other 2 patients had no treatment to the neck since this had been carried out previously. All the excised lymph nodes were examined and the histology was : $\mathrm{pN}$ - in 10 cases (37\%), $\mathrm{pN}+$ in 17 cases (63\%).

The 29 patients' follow-up has been from 6 to 37 months (mean 16 months and median 15.5; range: 6-37 months). At present 12 patients have no evidence of dis-
Table 3: Follow-up (6-37 months)

\begin{tabular}{ccc}
\hline & No. of patients & $\%$ \\
\hline NED & 12 & \\
AWSP & 2 & $41.3 \%$ \\
DOD & 7 & $6.7 \%$ \\
DOSP & 6 & $24.1 \%$ \\
DOC & 2 & $20.7 \%$ \\
& & $6.7 \%$ \\
\hline
\end{tabular}

NED No evidence of disease AWSP Alive with secondary primary DOD Dead of disease DOSP Dead of second primary DOC Dead of other causes

ease, 15 patients have died, 7 for his tumour, 6 for a second tumour, 2 for other causes, 2 are alive with second primary (oropharyngeal and lung) disease. Concerning the patients died for first tumor 3 died for lung metastases, 2 had a recurrence on $\mathrm{T}$ and 2 on $\mathrm{N}$. (Table 3 ).

\section{Discussion}

The poor prognosis of hypopharyngeal cancers compels the surgeon to choose a technique which allows wide surgical resection associated with a reconstruction procedure resulting in prompt restoration of physiological functions, low morbidity and a short period of hospitalisation. Total circular pharyngolaryngectomy has certainly made some impact on the prognosis, but above all on the quality of life of these patients $[15,18,19]$.

The surgical options for circumferential reconstruction of the hypopharynx include, besides the free flaps, myocutaneous pedicled flaps and visceral transpositions. The myocutaneous flaps have the disadvantage of being prone to local complications such as an high rate of salivary fistulae and dysphagia, difficult to treat because of the thickness of the flap [20]. Visceral transpositions have a higher perioperative morbidity and mortality $[11,21]$.

As far as concerns the free flaps, the fascio-cutaneous ones (forearm or lateral thigh) have the advantage of avoiding abdominal surgery. However, in order to tubulize the forearm flap, a big dimension of volar surface area is needed, causing a very poor aestethic results. The lateral thigh is not a first choice flap because is provided of a small vascular pedicles with an high thrombosis risk. In addition both flaps are prone to fistula formation because of the difficult adaptability of the mucous-cutaneous sutures [22-25].

The revascularized free flap of jejunum has been shown to be a flap with anatomo-physiological features suitable for the reconstruction of the hypopharyngo-oesophageal 
tract. The advantages are: one surgical session; fast recovery of physiological alimentation; possibility of reconstructing large defects; possibility of being performed even in patients previously treated with radiotherapy; good capacity to resist possible complementary radiotherapy [26].

Our surgical technique is quite similar to that describe by Julieron et al. [10] et Reece et al [27] The main difference with the first author is only the monitoring technique, in that we used an external monitor as Reece et al. and not the endoscopic vision. With this kind of surgical technique, the percentage of survival of jejunal loop flaps $(90 \%)$ and of minor complications such as fistulae or skin dehiscence $(1.5 \%)$ in our series is in line with that reported in the literature $[10,12,13,27]$. The survival of the loop was sustained even after complementary radiotherapy.

Concerning the functional results, good swallowing has been preserved in all our patients, none of whom has had to change from a normal diet. We have not recorded any problems with gastric reflux, in contrast to reports from other authors [13], perhaps because of the meticulous care taken during the extirpation to preserve the upper oesophageal sphincter and during the reconstruction to position the jejunal loop in an isoperistaltic direction $[12,13,21,27]$. No patient has a feeding tube and all patients are eating a reasonably normal diet with minimal dysphagia (3 patient for liquids, 1 for solids). The percentage of patients with dysphagia in our series is lower than that reported by other authors who have used revascularized skin flaps $[24,25]$. Only the 2 patients who had a second intention reconstruction with a flap of pectoralis major because of necrosis of the transplanted jejunal loop require periodic dilatations because of the thickness of the flap. Speech retraining after reconstruction with a jejunal loop is difficult. There are not many reports in the literature on speech restoration following reconstruction of the upper digestive tract using a free jejunum flap. This could be due either to the limited series of cases or to the unsatisfactory results, due to the lack of a muscular layer and/ or of a normal motor innervation in the jejunum graft wall $[15,27]$. All our patients where a phonatory prosthesis was positioned $(10 / 29)$ were able to achieve speech following speeching rehabilitation and all were satisfied with their own capacity to communicate. [29]

The hypopharyngeal cancer is a very poor prognosis tumor and our results are in line with the other authors where this kind of surgical technique was applied $[10,13,15,28]$. Our survival patient's percentage was $48.2 \%$ and 2 patients are alive with a disease or second neoplasm after a follow up period ranging from 6 to 37 months.

\section{Conclusions}

We assume that the indications of this kind of surgical technique are very strictly. The choice of the jejunum free flap depends both on the general conditions of the patient and on the extent of the cancer. Although the prognosis of these tumours (18-40\% at 5 years) remains poor, jejunum autografts are being shown to be an excellent choice for the reconstruction of the cervical hypopharyngooesophagus offering the patient quit rehabilitation and a reasonable quality of survival.

\section{List of abbreviations}

SQ: swallowing questionnaire

MRND: modified radical neck dissection

\section{Competing interests}

None declared.

\section{References}

I. Gluckman JL, Weissler MC, McCafferty G, et al: Partial vs. total esophagectomy for advanced carcinoma of the hypopharynx. Arch Otolaryngol Head Neck Surg 1987, I 1 3:69-72

2. Marzetti F, Ducci M, Pompei S, Marzetti A: Strategie ricostruttive nei tumori dell'ipofaringe e dell'esofago cervicale. In Atti Congresso Chirurgia ricostruttiva in oncologia cervico-cefalica Trieste 1996, 2II-223

3. Berger A, Tizian C, Hausamen J, Schulz-Coulon H, Lohlein D: Free jejunal graft for reconstruction of oral, oropharyngeal and pharyngoesophageal defects. J Reconstr Microsurg 1984, I:83-94

4. Stepnick DW, Hayden RE: Options for reconstruction of the pharyngoesophageal defect. Otolaryngologic Clinics of North America 1994, 27:|||5|-||58

5. Triboulet JP, Darras J, Bocquillon P, Ribiere P: La recostruction digestive après pharyngolaryngectomie totale circulaire. Chirurgie 1989, II 5:210-219

6. Peracchia A, Bardini R, Ruol A, Castoro E, Tiso E, Asolati M: Cancer de I'hypopharynx et de l'oesophage cervicale. Chirurgie 1990, 160:351-358

7. Avci C, Avtan L: La reconstruction de I'hypopharynx et l'oesophage cervical avec une greffe jéjunale libre à double pédicule. Chirurgie 1991, I 17:653-660

8. Haughey $\mathrm{BH}$ : The jejunal free flap in oral cavity and pharyngeal reconstruction. Otolaryngologic Clinics of North America 1994, 7:1159-1170

9. Yamamoto $Y$, Nohira K, Schintomi Y, Yoshida T, Minakawa H, Okushiba S, Fukuda S, Inuyama Y, Hosokawa M: Mesenteric flap in free jejunal transfer: a versatile technique for head and neck reconstruction. Head \& Neck. 1995, 17:213-218

10. Julieron M, Germain MA, Schwaab G, Marandas P, et al: Reconstruction par transplant libre de jéjunum après pharyngolaryngectomie totale circulaire. Ann Otolaryngol Chir Cervicofac. 1996 I I 3:269-275

II. Marks SC, Steiger Z: Combined pectoralis flap and gastric pullup for pharyngeal reconstruction. Head \& Neck. 1997, 19:134136

12. Bradford CR, Esclamado RM, Carrol WR, Sullivan MJ: Analysis of recurrence, complications, and functional results with free jejunal flaps. Head \& Neck. 1994, 16:149-154

13. Theile DR, Robinson DW, Theile DE, Coman WB: Free jejunal interposition reconstruction after pharyngolaryngectomy. Head \& Neck. 1995, I 7:83-88

14. Grimani L, Fossati GS, Benazzo M, Occhini A, et al: Hypopharynx reconstruction by a revascularized jejunum flap. Med Biol Environn. 1997, 25: 139-142

15. Julieron M, Germain MA, Schwaab G, Marandas P, et al: Reconstruction with free jejunal autograft after circumferential pharyngolaryngectomy: eighty-three cases. Ann Otol Rhinol Laryngol. 1998, 107 
16. Giovanoli P, Frey M, Schmid S, Flury R: Free jejunum transfer for functional reconstruction after tumour resection in the oral cavity and the pharynx: changes of morphology and function. Microsurgery. 1996, 17:535-44

17. German M, Uuureau J, Trotoux J, Agasson Voyeme AK: La reconstruction pharyngoesophagienne par transplant libre jéjunal revascularisé. Chirurgie. 1990, I1 6:78-88

18. Omura K, Misaki T, Watanabe $\mathrm{Y}$, Urayama H, Hashimoto T, Matsu T: Reconstruction with free jejunal autograft after pharyngolaryngoesophagectomy. Ann Thorac Surg. 1994, 96: I5-2I

19. Rénier S, Decroocq F, Simon C: Our experience in pharyngeal reconstruction with free flaps after total circular pharyngolaryngectomy. J Fr ORL. 1996, 45:2I-27

20. Fabian RL: Pectoralis major myocutaneous flap reconstruction of the laryngopharynx and cervical oesophagus. Laryngoscope. 1988, 98: 1277

21. Inoue $\mathrm{Y}$, Tai $\mathrm{Y}$, Fujita $\mathrm{H}$, Tanaka S, Migita $\mathrm{H}$, Kiyokawa $\mathrm{K}$, Hirano M, Kakegawa $T$ : A retrospective study of 66 esophageal reconstructions using microvascular anastomoses: problems and our methods for atypical cases. Plast Reconstr Surg. 1994, 94:285287

22. Hayden RE: Lateral cutaneous thigh flap. In microvascular reconstruction of the head and neck. Churchill Livingstone, New York. 1989, 2 I I228

23. Mayot D, Dron K, Moermann X, Lindas P, Perrin C: Our experience of pharyngeal reconstruction with forearm flap. J Fr ORL. 1993, 42:249-255

24. Stark B, Nathanson A: The free radial forearm flap: a reliable method for reconstruction of the laryngohypopharynx after in-continuity resection. Acta Otolaryngol (Stockh). 1998, I I 8:419422

25. Guler M, Isik S, Sezgin M: Pharyngoesophageal reconstruction with the tubed radial forearm free flap. Eur Arch Otorhinolaryngol. 1998, 255:24-26

26. Petruzzelli GJ, Johnson JT, Myers EN, Shestak K, Jones N, Cano E, Wagner $R$ : The effect of postoperative radiation therapy on pharyngoesophageal reconstruction with free jejunal interposition. Arch Otolaryngol Head Neck Surg. 1991, I 17 : 1265 - 1268

27. Reece GP, Bengston BP, Schusterman MA: Reconstruction of the cervical pharynx and cervical esophagus using free jejunal transfer. Clinics in Plastic Surgery. 1994, 21:125-136

28. Marzetti F: Pharyngoesophageal reconstruction after segmental resection by means of a double arteriovenous anastomosed free jejunal transplanted reservoir: a new and original surgical technique. Med Biol Environn. 1997, 25: 143-I47

29. Benazzo M, Bertino G, Lanza L, Occhini A, Mira E: Voice restoration after circumferential pharyngolaryngectomy with free jejunum repair. Eur Arch Otorhinolaryngol. 2001, 258:173-176

\section{Pre-publication history}

The pre-publication history for this paper can be accessed here:

http://www.biomedcentral.com/1471-2407/2/13/prepub
Publish with BioMed Central and every scientist can read your work free of charge

"BioMedcentral will be the most significant development for disseminating the results of biomedical research in our lifetime."

Paul Nurse, Director-General, Imperial Cancer Research Fund

Publish with BMC and your research papers will be:

- available free of charge to the entire biomedical community

- peer reviewed and published immediately upon acceptance

- cited in PubMed and archived on PubMed Central

- yours - you keep the copyright

Submit your manuscript here: BioMedcentral.com

http://www.biomedcentral.com/manuscript/ 